\title{
Low Wall Shear Stress Contributes to Atherosclerosis of the Carotid Artery in Hypertensive Patients
}

\author{
Yinong Jiang, Katsuhiko Kohara*, and Kunio Hiwada
}

\begin{abstract}
Numerous in vitro studies have indicated that low shear stress may contribute to intimal thickening and development of atherosclerosis. In the present study, we investigated wall shear stress in hypertensive patients and its relevance to atherosclerosis in the carotid arteries by means of a non-invasive technique. Fifty-five hypertensive patients and 23 normotensive controls were investigated. Intima-media thickness, number of plaques, internal dimension and blood flow velocity of the carotid artery were evaluated. Wall shear stress was calculated using the Poiseuillean parabolic model of velocity distribution as follows: shear stress $=4 \times$ blood viscosity $\times$ central line flow velocity/internal dimension. Hypertensive patients showed increased intima-media thickness and dilated common carotid arterial dimension relative to normotensive controls. There was no difference in blood viscosity between the two groups. Both the mean shear stress and systolic peak shear stress were significantly lower in hypertensive patients than normotensive controls. Further, wall shear stress at both mean and peak velocity was significantly and negatively related to intima-media thickness and number of plaques in hypertensive patients, as well as in the total study population. These findings indicate that structural and functional alterations in the common carotid artery of hypertensive patients further precipitates atherosclerosis through low shear stress. (Hypertens Res 1999; 22: 203-207)
\end{abstract}

Key Words: wall shear stress, carotid artery, intima-media thickness, plaque

Atherosclerosis is a systemic disease relevant to various risk factors including aging, hypertension, hyperlipidemia, diabetes mellitus, and smoking $(1-3)$. As a local mechanism for the progression of atherosclerosis, shear stress, or the frictional force exerted on the intimal surface of the artery by the circulating blood column, has been the focus of numerous in vitro studies. There is growing evidence that higher shear stress protects the development of atherosclerosis through various mechanisms, including improvement of endothelial function (4-6), reduction of adhesion molecules $(7,8)$, and enhancement of nitric oxide synthesis (9-11). By precisely determining the internal diameter and flow velocity, several clinical studies have determined shear stress of carotid artery $(12,13)$ and of radial artery (14). In one such study, Gnasso et al. (12) reported that increased intima-media thickness (IMT) was negatively correlated with shear stress in the carotid arteries of healthy male subjects.

In our previous study, we demonstrated that hypertensive patients showed increased IMT, dilatation of internal diameter and higher prevalence of plaques in the common carotid artery (CCA) (15). These findings indicate that shear stress in the carotid artery is reduced in hypertensive patients compared with normotensive controls. In the present study, we investigated the wall shear stress in hypertensive patients and its relevance to IMT in the CCA by means of a non-invasive technique.

\section{Subjects and Methods}

\section{Study Population}

Fifty-five hypertensive patients and 23 sex and agematched normotensive controls participated in this study. All hypertensive patients were untreated or had been free from medication for at least $1 \mathrm{wk}$ before the investigation. Blood pressure was determined by sphygmomanometer before Echo-Doppler examination. Informed consent for all procedures was obtained from each patient.

\section{Echo-Doppler Examination of Carotid Artery}

Carotid arteries were evaluated using SSD-2000 (Aloka Co., Ltd., Tokyo, Japan) and a $7.5-\mathrm{MHz}$ probe equipped with a Doppler system as previously described (15-18). In brief, subjects inclined their necks in a slight hyperextended position for determination of the optimal approach for visualization of the common carotid arteries. From multiple approaches, we detected the presence of discrete segments of atherosclerosis (plaque). Plaque was considered to exist on walls that were at least $50 \%$

From the Second Department of Internal Medicine, and *Department of Geriatric Medicine, Ehime University School of Medicine, Ehime, Japan.

Address for Reprints: Katsuhiko Kohara, M.D., Department of Geriatric Medicine, Shigenobu-cho, Onsen-gun, Ehime 791-0295, Japan.

Received Octorber 29, 1998. 
Table 1. Clinical Characteristics of Normotensive Controls and Hypertensive Patients

\begin{tabular}{lccc}
\hline & Normotensive controls & Hypertensive patients & $p$ \\
\hline$n$ (male/female) & $23(15 / 8)$ & $55(38 / 17)$ & NS \\
Age $(\mathrm{yr})$ & $57 \pm 11$ & $59 \pm 11$ & $\mathrm{NS}$ \\
Body mass index $\left(\mathrm{kg} / \mathrm{m}^{2}\right)$ & $22.6 \pm 3.9$ & $25.5 \pm 3.0$ & 0.001 \\
Systolic blood pressure $(\mathrm{mmHg})$ & $123 \pm 10$ & $156 \pm 20$ & 0.0001 \\
Diastolic blood pressure $(\mathrm{mmHg})$ & $77 \pm 7$ & $90 \pm 12$ & 0.0001 \\
Hyperlipidemia $(\%)$ & 35 & 39 & $\mathrm{NS}$ \\
Diabetes $(\%)$ & 17 & 30 & $\mathrm{NS}$ \\
\hline
\end{tabular}

Values are mean \pm SD. NS: not significant.

more thick than the surrounding area. IMT of the far wall was measured in the right common carotid artery at 1 and $2 \mathrm{~cm}$ proximal to the bulb from the anterior, lateral, and posterior approaches, then averaged to obtain the mean IMT. Measurements were never taken at the level of a discrete plaque. Hemodynamically significant luminal stenosis $(\geq 50 \%)$ was not detected in any subjects.

Two-dimensionally guided M-mode tracings of the right common carotid artery at $1 \mathrm{~cm}$ proximal to the bulb were recorded simultaneously by electrocardiograph and phonocardiograph. M-mode images were obtained in real time using a frame grabber. The axial resolution of the M-mode system was 0.1 $\mathrm{mm}$. End-diastolic (IDd) and peak-systolic internal dimensions (IDs) were obtained by continuous tracing of the intimal-luminal interface of the near and far wall of the CCA in three cycles and averaged.

Doppler evaluation was performed on the right CCA at the same site. The carotid artery was scanned in the anterior projection. Under guidance of color flow mapping, the sample volume was located at the center of the vessel. Peak systolic and diastolic velocities (Vs and Vd, respectively) were determined from blood flow wave measurements at three cardiac cycles and averaged. Mean velocity ( Vm) was obtained by electronic integration of the instantaneous flow velocity curves.

\section{Wall Shear Stress}

In the present study, we evaluated the peak and mean wall shear stresses as the highest and mean shear stresses, respectively. On the day of the echoDoppler examination, blood was withdrawn and heparinized for the determination of blood viscosity. The viscosity $(\eta)$ was measured by a cone/plate viscometer (Biorheolizer TOKIMEC, Osaka, Japan) at the shear rate of $375 / \mathrm{s}$. Wall shear rates were calculated with a Poiseuillean parabolic model of velocity distribution across the arterial lumen, according to the following formulas $(12,13)$ :

$$
\begin{aligned}
& \gamma \mathrm{s}=4 \mathrm{Vs} / \mathrm{IDs} \\
& \gamma \mathrm{m}=4 \mathrm{Vm} / \mathrm{IDd},
\end{aligned}
$$

where $\gamma \mathrm{s}$ and $\gamma \mathrm{m}$ represent peak systolic shear rate and mean shear rate, respectively. Peak ( $\tau$ p) and mean wall shear stress $(\tau \mathrm{m})$ were calculated by the following formulas under assumption that blood is Newtonian fluid $(12,13)$ :

$$
\begin{aligned}
& \tau \mathrm{p}=\eta \cdot \gamma \mathrm{s} \\
& \tau \mathrm{m}=\eta \cdot \gamma \mathrm{m} .
\end{aligned}
$$

\section{Statistical Analysis}

All values are expressed as mean \pm SD. Differences in all measurements between normotensive and hypertensive subjects were assessed by the Student's $t$ test. Differences in the prevalence of hyperlipidemia and diabetes were analyzed by $\chi^{2}$ test. Pearson's correlation coefficient was used to test the association between all parameters. A probability of less than 0.05 was defined as statistically significant.

\section{Results}

\section{Characteristics of Study Subjects and Geometry of $C C A$}

Table 1 summarizes the clinical profiles of normotensive controls and hypertensive patients. With the exceptions of body mass index and hypertension, there were no differences in risk factors between the two groups. Structural and functional parameters for the shear stress are summarized in Table 2. Hypertensive patients showed increased IMT and dilated carotid arterial dimension relative to normotensive controls. There was no difference in blood viscosity between the two groups. The mean shear stress and peak systolic shear stress were significantly lower in hypertensive patients than in normotensive controls.

\section{Carotid Atherosclerosis and Wall Shear Stress}

Figure 1 shows the relationships between mean and peak wall shear stress and IMT. Both mean and peak wall shear stresses were inversely and significantly related to IMT in this study population. Figure 2 illustrates the relationship between mean and peak wall shear stress and number of plaques. There was also a significant negative correlation between number of plaques and both mean shear stress and peak systolic stress.

\section{Discussion}

It has been postulated that low shear stress contributes to intimal thickening and atherosclerotic development $(19,20)$. In the present study, we evaluated wall shear stress in vivo using the Poiseuillean parabolic model of blood velocity distribution (12). The findings that hypertensive patients had dilated carotid arterial dimension and slow blood flow velocity were consistent with our previous findings (15). Although there was no significant difference in 
Table 2. Geometry and Functional Parameters of the Carotid Artery in Normotensive Controls and Hypertensive Patients

\begin{tabular}{lccc}
\hline & Normotensive controls & Hypertensive patients & $p$ \\
\hline Intima-media thickness $(\mathrm{mm})$ & $0.74 \pm 0.11$ & $0.89 \pm 0.16$ & 0.0002 \\
Plaque $(n)$ & $0.09 \pm 0.29$ & $0.82 \pm 1.12$ & 0.003 \\
Systolic carotid dimension $(\mathrm{mm})$ & $6.8 \pm 0.6$ & $7.5 \pm 0.9$ & 0.0007 \\
Diastolic carotid dimension $(\mathrm{mm})$ & $6.5 \pm 0.7$ & $7.1 \pm 0.9$ & 0.0016 \\
Viscosity of blood (centipoise) & $4.1 \pm 0.6$ & $4.1 \pm 0.7$ & $\mathrm{NS}$ \\
Systolic peak velocity $(\mathrm{cm} / \mathrm{s})$ & $82.4 \pm 16.3$ & $73.5 \pm 18.1$ & 0.046 \\
Mean velocity $(\mathrm{cm} / \mathrm{s})$ & $43.2 \pm 8.9$ & $37.2 \pm 11.0$ & 0.025 \\
Peak shear stress $\left(\right.$ dyne $\left./ \mathrm{cm}^{2}\right)$ & $20.1 \pm 5.4$ & $16.4 \pm 5.7$ & 0.0095 \\
Mean shear stress $\left(\right.$ dyne $\left./ \mathrm{cm}^{2}\right)$ & $11.0 \pm 3.1$ & $8.7 \pm 3.5$ & 0.0069 \\
\hline
\end{tabular}

Values are mean \pm SD. NS: not significant.
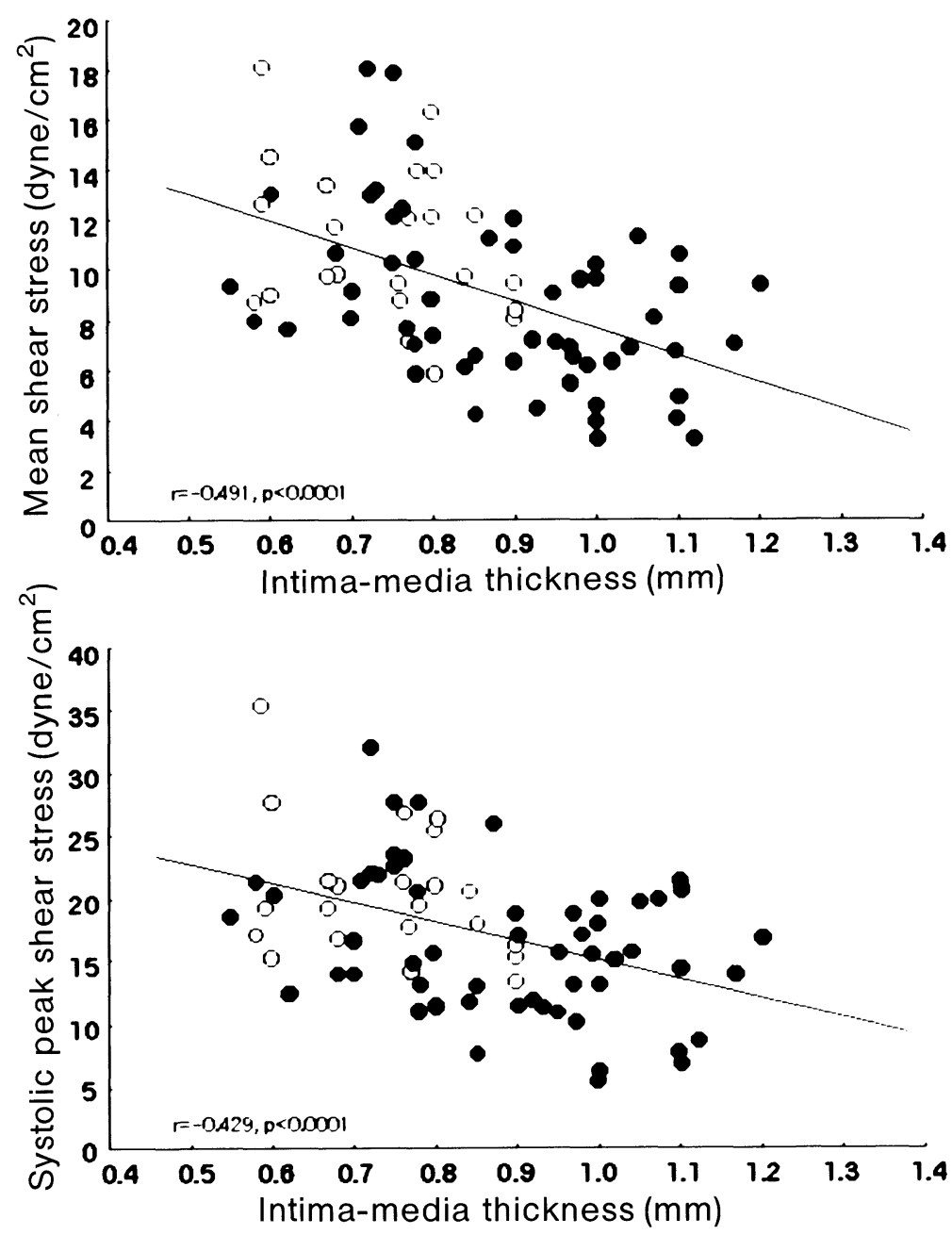

Fig. 1. The relations between mean shear stress (upper) or systolic peak shear stress (bottom) and intima-media thickness in the total population of normotensive controls $(\bigcirc)$ and hypertensive patients (O).

the blood viscosity between hypertensive patients and normotensive controls, this study was the first to demonstrate that hypertensive patients had a significantly reduced wall shear stress in either systolic peak phase, as well as mean cardiac cycle.

To further investigate the possibilities of a direct relationship between local shear stress and carotid arterial atherosclerosis, we evaluated correlation between local shear stress and mean IMT and number of plaques. The results showed that the wall shear stress at both mean and peak velocity was significantly and negatively related to atherosclerosis in 

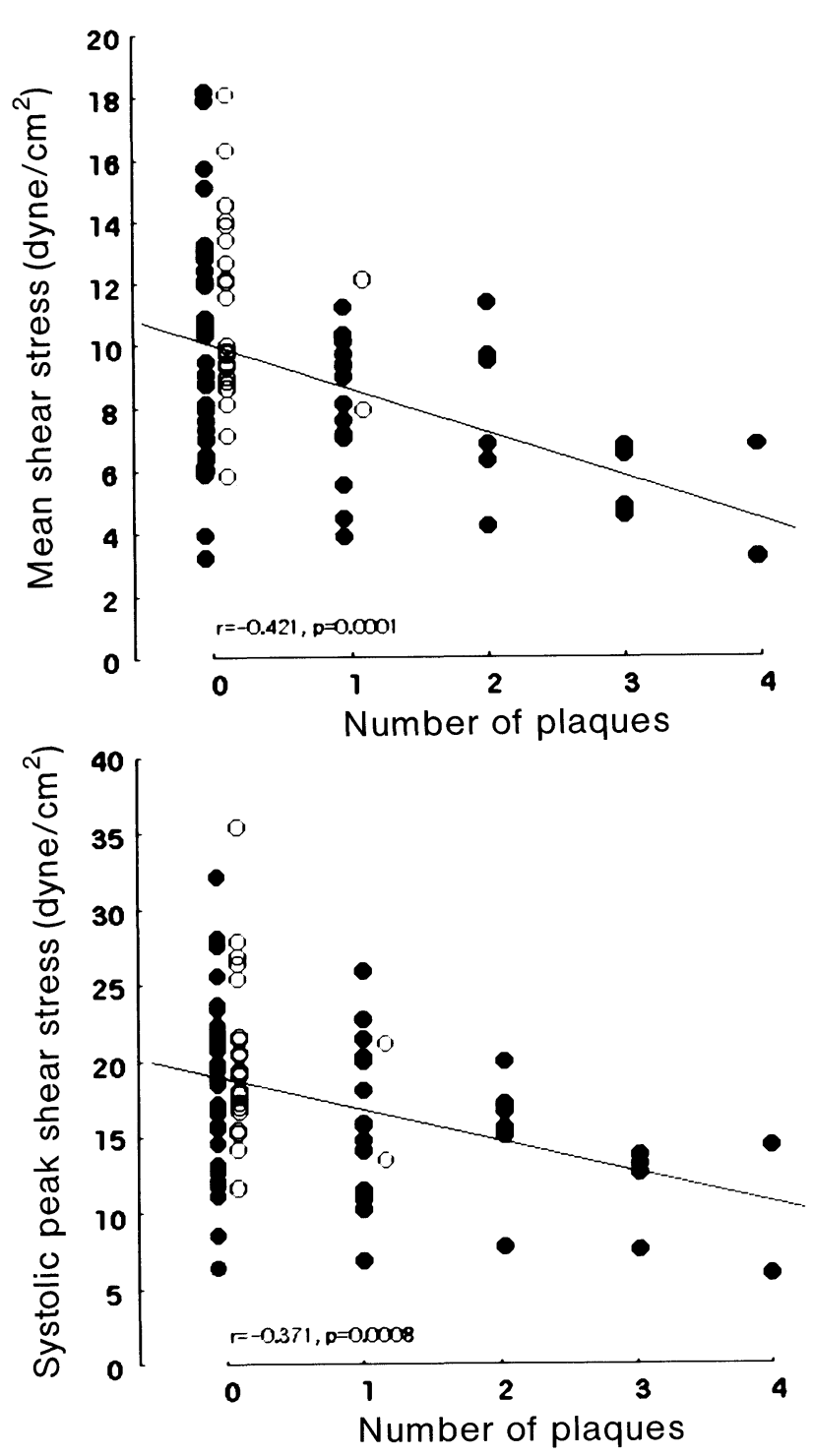

Fig. 2. The relations between mean shear stress (upper) or systolic peak shear stress (bottom) and number of plaques in the total population of normotensive controls $(\bigcirc)$ and hypertensive patients

the carotid artery. A significantly negative association between IMT and wall shear stress in the CCA of normotensive subjects has also been reported in normal healthy subjects (12). Our study confirmed a similar relationship in hypertensive patients.

It has been suggested that low shear stress may contribute to an increase in fluid residence time, which in turn may result in increased transport of platelets, macrophages, and other atherosclerotic particles $(21,22)$. It has been demonstrated that higher shear stress can inhibit the adhesion of lymphocytes to endothelial cells by reducing the expression of adhesion molecules (7). In addition, recent studies have suggested that shear stress modulates the transcription of genes for nitric oxide synthase, platelet-derived growth factor (PDGF) A- and B-chain, prostacyclins, and transforming growth factor $\beta_{1}(9-11,23)$. The change of these substances induced by low shear stress could contribute to the progression of vascular remodeling.

In conclusion, our study demonstrated that local wall shear stress is inversely related to IMT and number of plaques of the CCA. These findings indicate that structural and functional alterations in the common carotid artery might advance the process of atherosclerosis through low shear stress in hypertensive patients.

\section{References}

1. Kamide K, Rakugi H, Nagano $\mathrm{M}$, et al: Influence of aging on progression of cardiovascular complications associated with insulin resistance in patients with essential hypertension. Hypertens Res 1997; 20: 127132.

2. Kawamori R: Insulin resistance seen in non-insulin dependent diabetes mellitus and hypertension. Hypertens Res 1996; 19 (Suppl I): S61-S64.

3. Kohara $\mathrm{K}$, Igase $\mathrm{M}$, Jiang $\mathrm{YN}$, et al: Asymptomatic cerebrovascular damages in essential hypertension in the elderly. Am J Hypertens 1997; 10: 829-835.

4. Cooke JP, Rossitch E Jr, Andon NA, Loscalzo J, Dzau VJ: Flow activates an endothelial potassium channel to release an endogenous nitrovasodilator. $J$ Clin Invest 1991; 88: 1663-1671.

5. Harrison VJ, Ziegler T, Bouzourene K, Suciu A, Silacci P, Hayoz D: Endothelin-1 and endothelinconverting enzyme-1 gene regulation by shear stress and flow-induced pressure. J Cardiovasc Pharmacol 1998; 31 (Suppl 1): S38-S41.

6. Rieder MJ, Carmona R, Krieger JE, Pritchard KA $\mathrm{Jr}$, Greene AS: Suppression of angiotensin-converting enzyme expression and activity by shear stress. Circ Res 1997; 80: 312-319.

7. Ando J, Tsuboi H, Korenaga R, et al: Shear stress inhibits adhesion of cultured mouse endothelial cells to lymphocytes by downregulating VCAM-1 expression. Am J Physiol 1994; 267: C679-C687.

8. Gerszten RE, Lim Y-C, Ding HT, et al: Adhesion of monocytes to vascular cell adhesion molecule-1-transduced human endothelial cells: implications for atherogenesis. Circ Res 1998; 82: 871-878.

9. Zhu Z-G, Li H-H, Zhang B-R: Expression of endothelin-1 and constitutional nitric oxide synthase messenger RNA in saphenous vein endothelial cells exposed to arterial flow shear stress. Ann Thorac Surg 1997; 64: 1333-1338.

10. Matrougui K, Maclouf J, Lévy BI, Henrion D: Impaired nitric oxide- and prostaglandin-mediated responses to flow in resistance arteries of hypertensive rats. Hypertension 1997; 30: 942-947.

11. Noris M, Morigi M, Donadelli R, et al: Nitric oxide synthesis by cultured endothelial cells is modulated by flow conditions. Circ Res 1995; 76: 536-543.

12. Gnasso A, Carallo C, Irace C, et al: Association betwèen intima-media thickness and wall shear stress in common carotid arteries in healthy male subjects. Circulation 1996; 94: 3257-3262.

13. Gnasso A, Irace $\mathrm{C}$, Carallo $\mathrm{C}$, et al: In vivo association between low wall shear stress and plaque in subjects with asymmetrical carotid atherosclerosis. Stroke 1997; 28: 993-998.

14. Girerd X, London G, Boutouyrie P, Mourad J-J, Safar M, Laurent S: Remodeling of the radial artery in response to a chronic increase in shear stress. 
Hypertension 1996; 27: 799-803.

15. Jiang YN, Kohara K, Hiwada K: Alteration of carotid circulation in essential hypertensive patients with left ventricular hypertrophy. J Hum Hypertens 1998; 12: $173-179$

16. Fukudome Y, Fujii K, Abe I, et al: Ultrasonographic assessment of regional differences in atherosclerotic lesions in patients with hypertension, diabetes mellitus, or both. Hypertens Res 1997; 20: 175-181.

17. Lemne C, Jogestrand T, de Faire U: Carotid intimamedia thickness and plaque in borderline hypertension. Stroke 1995; 26: 34-39.

18. Roman MJ, Pickering TG, Pini R, Schwartz JE, Devereux RB: Prevalence and determinants of cardiac and vascular hypertrophy in hypertension. Hypertension 1995; 26: 369-373.

19. Zarins CK, Giddens DP, Bharadvaj BK, Sottiurai VS, Mabon RF, Glagov S: Carotid bifurcation athero- sclerosis: quantitative correlation of plaque localization with flow velocity profiles and wall shear stress. Circ Res 1983; 53: 502-514.

20. Asakura T, Karino T: Flow patterns and spatial distribution of atherosclerotic lesions in human coronary arteries. Circ Res 1990; 66: 1045-1066.

21. Zarins CK, Bomberger RA, Glagov S: Local effects of stenoses: increased flow velocity inhibits atherogenesis. Circulation 1981; 64 (Suppl II): II-221-II227.

22. Dobrin PB, Littooy FN, Endean ED: Mechanical factors predisposing to intimal hyperplasia and medial thickening in autogenous vein grafts. Surgery 1989; 105: 393-400.

23. Malek AM, Izumo S: Molecular aspects of signal transduction of shear stress in the endothelial cell. $J$ Hypertens 1994; 12: 989-999. 